prevent hospitalizations and emergency visits. In addition, ALAI is well tolerated, achieving a high percentage of patients in monotherapy.

65

\section{A Retrospective Study of Aripiprazole Long- acting Once-Monthly Introduction Patterns in Galicia}

Rebeca Méndez Iglesias, $M D^{\prime}$; and Daniel Núñez Arias, $M D^{2}$

${ }^{1}$ Psychiatrist, Department of adult psychiatry,

A Coruña Hospital, Spain

${ }^{2}$ Psychiatrist, Department of adult psychiatry, Ferrol

Hospital, Spain

Águeda Fernández Quintana, MD: Psychiatrist, Department of adult psychiatry, Västmanl and Hospital, Sweden.

ABSTRACT: Background: Antipsychotic drug treatment is a key component of multiple psychiatric treatment algorithms. Second-generation long-acting injectable antipsychotics (LAIs) have been shown to improve adherence in numerous clinical trials. Patients who can benefit from LAIs therapy can be treated with aripiprazole long acting once-monthly (AOM). However the nature of the introduction patterns of AOM is not well characterized in clinical practice.

METHODS: A retrospective observational study of AOM introduction was conducted on 157 patients aged 18-75 years-old (95 males, 62 females) who were initiated on AOM treatment between January 2017 December 2017 in two independent Mental Health Units in the autonomous region of Galicia (Spain). An analysis of the different trends in switching strategies and its adaptation to the prescribing information was carried out. Results were compared between different dose treatment plans and a comparison between inpatients and outpatients' outcomes was also undertaken. Additional data regarding off-label use was obtained from the sample.

RESULTS: The sample was composed of 157 patients: $31 \%$ diagnosed of Schizophrenia $(\mathrm{n}=48), 14 \%$ Schizoaffective Disorder $(\mathrm{n}=22), 21 \%$ Delusional Disorder $(\mathrm{n}=33)$, 17\% Bipolar Disorder $(n=27), 10 \%$ Brief Psychotic Disorder $(\mathrm{n}=15), 4 \%$ Psychotic Disorder Not Specified $(n=6), 2 \%$ Obsessive-Compulsive Disorder $(n=3), 2 \%$ Paranoid Personality Disorder $(n=3)$. Regarding the location of the first dose administration: 44\% $(n=69)$ were administered in an Acute Psychiatric Inpatient Unit, 44\% ( $\mathrm{n}=65)$ were administered in Mental Health Outpatient Clinics, 11\% $(\mathrm{n}=18)$ in Psychiatric Day
Hospitals and $3 \%(\mathrm{n}=5)$, in Assertive Community Treatment Programs. 74\% $(\mathrm{n}=116)$ of patients received an initial dose of $400 \mathrm{mg}$ of AOM whereas $26 \%(\mathrm{n}=41)$ were given $300 \mathrm{mg}$ of AOM. The previous antipsychotic was aripiprazole orale $(\mathrm{OA})$ in $61 \%(n=96)$ of the cases. The most frequent switch between LAIs was "immediate switch" and in the switch between orale antipsychotics and AOM "tapering and overlap" was found to be the most common pattern. The average dose was $20 \mathrm{mg} /$ day in all groups except for patients diagnosed with Delusional Disorder (15 mg/day). The average duration of treatment with OA after the first dose was: 32 days for patients with Schizophrenia, 23 days for Delusional Disorder, 30 days for Bipolar Disorder and 19 days in Schizoaffective Disorder.

CONCLUSIONS: Our analysis identified two main patterns of drug switching, the most frequent being "tapering and overlap" in oral treatment, followed by "immediate switch" in patients treated with LAIs.

Although our patients are unlike many of those enrolled in clinical trials, the present study indicates that the predominant switching strategies conforms with the Safety Data Sheet.

\section{7}

Effects of Long-term Valbenazine on Psychiatric Status in Patients with Tardive Dyskinesia and a Primary Mood Disorder

Roger S. McIntyre, M.D.; FRCPC'; Gary Remington, MD, PhD, FRCP ${ }^{2}$; Christoph U. Correll, $M D^{3}$; Rachel Weber, PhD ${ }^{4}$; Khodayar Farahmand, Pharm ${ }^{5}$; Leslie Lund, $M D^{6}$; Joshua Burke, $M S^{7}$; and Scott Siegert, PharmD ${ }^{8}$

${ }^{1}$ Professor of Psychiatry and Pharmacology, Department of Psychiatry, University of Toronto; Toronto, Canada

${ }^{2}$ Senior Scientist in the Campbell Family Mental Health Research Institute and Chief of the Schizophrenia Division, Centre for Addiction and Mental Health; Toronto, Ontario, Canada

${ }^{3}$ Medical Director, Recognition and Prevention (RAP) Program, The Zucker Hillside Hospital, Glen Oaks, NY and Professor of Psychiatry and Molecular Medicine, The Donald and Barbara Zucker School of Medicine at Hofstra/Northwell, Hempstead, NY

${ }^{4}$ Medical Communications Specialist, Neurocrine Biosciences, Inc., San Diego, CA

${ }^{5}$ Director, Medical Communications, Neurocrine Biosciences, Inc., San Diego, CA

${ }^{6}$ Medical Director, Neurocrine Biosciences, Inc., San

Diego, CA

${ }^{7}$ Director, Biostatistics and Data Management, Neurocrine Biosciences, Inc., San Diego, CA 
${ }^{8}$ Executive Director, Medical Affairs, Neurocrine Biosciences, Inc., San Diego, CA

ABSTRACT: Objective: Valbenazine is approved for tardive dyskinesia (TD) in adults based on clinical trials that included patients with mood disorders (e.g., bipolar disorder, major depressive disorder). In two longtermphase 3 trials, KINECT 3 (NCT02274558) and KINECT 4 (NCT02405091), sustained TD improvements were found in participants who received oncedaily treatment with valbenazine $(40$ or $80 \mathrm{mg}$ ). Data from these studies were analyzed post hoc to evaluate changes in psychiatric status of patients with a primary mood disorder.

METHODS: Data were pooled from participants with mood disorders in KINECT 3 (6-week double-blind, placebocontrolled period; 42-week double-blind extension period; 4-week drug-free washout) and KINECT 4 (48 week open-label treatment; 4-week drug-free washout). At screening, patients must have had a Brief Psychiatric Rating Scale total score $<50$. Mood changes were evaluated after long-term treatment (Week 48) and washout (Week 52) using the Young Mania Rating Scale (YMRS) and Montgomery-Åsberg Depression Rating Scale (MADRS). For each scale, mean changes from baseline in the total score and individual item scores were analyzed descriptively.

RESULTS: Of the 95 participants with a primary mood disorder $(40 \mathrm{mg}, \mathrm{n}=32 ; 80 \mathrm{mg}, \mathrm{n}=63), 59$ (62.1\%) were diagnosed with bipolar disorder, $32(33.7 \%)$ with major depressive disorder, and 4 (4.2\%) with another mood disorder. A majority of all mood participants received concomitant antidepressants $(84.2 \%)$ and/or antipsychotics $(76.8 \%)$ during treatment; other common concomitant medications included antiepileptics (47.4\%), anxiolytics (38.9\%), and anticholinergics (22.1\%). Mean YMRS and MADRS total scores in all mood participants indicated mood symptom stability at baseline (YMRS, 2.7; MADRS, 5.9). This stability was maintained during the studies, as indicated by minimal changes from baseline in mean total scores (YMRS: Week 48, 1.0; Week 52, -1.0; MADRS: Week 48, 0.3; Week 52, 0.9). Changes in individual items on both scales were also small $(< \pm 0.3)$, indicating no clinically significant changes or worsening in specific mood symptoms or domains.

CONCLUSIONS: Mood symptom stability was maintained in patients with TD and a primary mood disorder who received up to 48 weeks of treatment with oncedaily valbenazine in addition to their psychiatric medication(s).

Funding Acknowledgements: Neurocrine Biosciences, Inc.
68

Cognitive Impairment in Schizophrenia. Firstgeneration Long-acting Antipsychotics versus Aripiprazole Long-acting Injectable

Samuel-Leopoldo Romero Guillena, $\mathrm{MD}^{\prime}$; and BeatrizOda Plasencia García de Diego, $M D^{2}$

${ }^{1}$ Department of Psychiatry, UGC Salud Mental, Macarena Hospital, Seville, Spain

${ }^{2}$ Department of Psychiatry, De la Merced Hopsital, Osuna, Seville, Spain

ABSTRACT: Study Objectives: To assess differences in cognitive impairment in a group of patients with schizophrenia receiving first-generation long-acting antipsychotics (FG-LAI) versus Aripiprazole long-actinginjectable (ALAI).

METHOD: A descriptive, cross-sectional, multicenter study.

Study sample: 28 outpatients with stable schizophrenia (18 men and 10 women) with ages ranging from 22 to 64 years.

Inclusion criteria were: Clinically stable patients with a diagnosis of schizophrenia (according to DSM-5 criteria) and without any changes to their antipsychotic or antidepressant therapy in the last six months.

Simple stratified sampling was performed to collect data from patients with schizophrenia receiving FG-LAI $(\mathrm{n}=14)$ versus patients with schizophrenia receiving Aripiprazole long-acting injectable (ALAI) $(n=14)$

Groups were matched by age, gender, years of evolution of the disease, and years on formal education.

Functionality in the different cognitive domains was evaluated based on the Brief Assessment of cognition in Schizophrenia (BACS.), a hetero-applied instrument, which Spanish version has been validated. BACS evaluates the following cognitive domains:

Verbal memory (V.M): Word list test

Working memory (W.M): Digit sequencing task

Motor speed (M.S): Token motor task

Verbal fluency (V.F): Semantic or category fluency

Attention (A): Symbol coding

Executive function (E.F): Tower of London

The data obtained were analyzed using the SPSS 22.0 statistical package Differences between the means of quantitative variables were assessed using the independent-sample Student-t test. Individual test scores were converted into standardized ( $\mathrm{T}$ and $\mathrm{Z}$ ) scores and composite scores that were corrected for age and gender. Informed consent was obtained from all patients according to the Declaration of Helsinki (World Medical Association).

RESULTS: In the group receiving ALAI, the most severely impaired cognitive domain was attention, whereas motor 